\title{
Multi-jet gas cooling of in-beam foils or specimens: CFD predictions of the convective heat-transfer coefficient
}

\author{
Gideon Steyn ${ }^{1, *}$, Christiaan Vermeulen ${ }^{2, * *}$, and Michael Steyn ${ }^{3}$ \\ ${ }^{1}$ iThemba LABS, National Research Foundation, P.O. Box 722, Somerset West, 7129, South Africa \\ ${ }^{2}$ Isotope Program, Los Alamos National Laboratory, Los Alamos, NM 87545, USA \\ ${ }^{3}$ Stellenbosch University, Matieland, 7602, South Africa
}

\begin{abstract}
An experiment was designed to investigate the possible enhancement of the convective heattransfer coefficient by utilizing multiple, parallel jets in the cooling of a small heated surface, such as typically induced by an accelerated ion beam on a thin foil or specimen. The hot spot was provided using a small electrically heated plate. It was found that heat-transfer calculations by means of simple empirical methods based on dimensional analysis are not useful in this case and that advanced computational fluid dynamics (CFD) mod-elling is essential to interpret the results. It is shown that enhanced convective cooling can indeed be obtained with a multi-jet configuration as compared to a single-jet configuration but only under very selective conditions. An improperly designed multi-jet configuration can also provide significantly reduced cooling relative to the single-jet case and the estimation of the behavior of any particular jet geometry is eminently non-intuitive. CFD provides acceptable quantitative results and seem to be the only tool available to gain an understanding of these complex flows where simple models and "rules of thumb" cannot be relied upon. - LA-UR-18-29455
\end{abstract}

\section{Introduction}

In a previous study (presented at INTDS 2016, see Ref. [1]), the focus was on the use of a single jet of gas in the convective cooling of thin in-beam materials, such as metal foils used as beam windows in a radionuclide production target station. Here we present a continuation of that work, where the investigation has been extended to multi-jet cooling of similar objects. This paper should therefore be read in conjunction with our previous work [1] to follow the full development of the topic. Of particular interest is the double-foil window concept, which is often employed to isolate the beamline vacuum from target materials. Such beam exit windows normally consist of two closely spaced foils through which a gas (e.g. helium) is circulated at a high flow rate to remove the heat induced by the beam.

What we are primarily interested in is the convective heat transfer from a small heated surface where an accelerated ion beam impinges on a target material. It is important to be clear on what is meant by "small heated surface" or "small hot spot" in the context of this study. There are many examples in the literature where an incident beam of ions generate a "small" heated area and one can easily familiarize with such situations, however, from a fluid dynamics perspective one should see the dimensions of a "small object" in relation to the dimensions of the inlet and exhaust ports that deliver and remove the coolant. When the heated area has similar or smaller dimensions relative

\footnotetext{
*e-mail: deon@tlabs.ac.za

**e-mail: etienne@lanl.gov
}

to those of the coolant ports, it is appropriate to speak of a "small hot spot".

The idea of using multiple jets in the heat transfer from a small hot spot is in a certain sense an anomaly as multijet cooling had been introduced for the effective cooling of large heated surfaces. Typically, high-intensity heat transfer from a large surface area is achieved by means of an array of closely-spaced gas jets (usually air) impinging perpendicularly to the surface [2-4], such as in the drying of paper and textiles, tempering of glass, annealing of metal and plastic sheets, cooling of turbojet engine structures, etc. Examples in the literature are numerous and diverse. A common aspect is that these heated surfaces are typically very large in comparison to the jet diameter. Why, then, should one be interested in an investigation on multijet cooling of small heated surfaces?

The answer relates to a particular result reported in the literature by Chang et al. [5], where an enhanced convective heat-transfer coefficient was found with a 25 -jet configuration $(5 \times 5$ matrix $)$ as compared to their single-jet measurements in a relatively small experimental assembly. These authors interpreted their result as due to an increased turbulence caused by the interaction of neighboring impinging jets, resulting in a higher average heat transfer, even though the local heat-transfer coefficients at the jet stagnation points were lower than in the single-jet case. Since the formalism by Chang and co-workers works well in the case of single-jet helium cooling of double-foil windows (our previous study - see Ref. [1]), we were curious about their multi-jet formalism and its possible application in convective cooling predictions for beam windows. 
Many strategies are reported in the literature to enhance turbulence. So-called "passive turbulence promoters" are objects placed upstream from the nozzle exit, e.g. twisted tapes to cause swirl flow [6,7], nozzle modifiers such as triangular tabs [8], etc. In the present study, the interaction of adjacent jets as a means to enhance turbulence has been investigated. We were primarily interested to see if the addition of a simple multiple-hole orifice plate could be used as a multi-jet nozzle in order to increase the turbulence, hence the overall heat-transfer coefficient.

At the 2016 INTDS Conference, we presented a paper on single-jet gas cooling of beam windows [1] in which we pointed out that certain empirical relations based on dimensional analysis have good predictive power. In addition, more advanced modelling based on computational fluid dynamics (CFD) proved u seful to gain a better understanding of the turbulence and heat transfer inside such beam window assemblies. We also presented an experimental set-up designed to measure convective heattransfer coefficients with a single gas jet. At that same time, we also had in our possession a set of measured data for multi-jet impingement heat transfer, which we did not present at INTDS 2016 because we did not then knew how to interpret those results. We are now in a better position to present those measurements as well as corresponding CFD simulations to assist with the interpretation.

In Sec. 2, the use of empirical relations based on dimensional analysis are briefly $d$ iscussed $f$ or the $c$ ase of multi-jet cooling. A description of the experimental setup is presented in Sec. 3 as well as the new experimental data. Aspects of the CFD study are presented in Sec. 4, followed by some comments in conclusion in Sec. 5 .

\section{Dimensional analysis in multi-jet heat transfer - empirical formalisms}

Many authors attempted to find appropriate empirical expressions for multi-jet heat transfer in terms of the Reynolds number, Nusselt number, Prandtl number, the geometric variables such as jet spacing, nozzle diameter, jet-to-surface spacing and the relevant fluid flow parameters. It transpired that success was rather limited and confined to very specific cases. There were simply too many correlations between too many variables, thus a general description proved to be elusive.

In our view, a seminal study was published in 1970 by Kercher and Tabakoff [2]. These authors employed the Buckingham pi theorem, which is the underlying the-orem in a whole range of heat-transfer expressions, such as the Dittus-Boelter, Sieder-Tate and Petukhov-Kirillov equations (see ref. [1] and references therein). Kercher and Tabakoff explored correlations of the following type in conjunction with measured heat-transfer data, employing a square array of round jets of the coolant (using forced convection of air in that particular study):

$$
\mathrm{Nu}_{D}=\theta_{1} \operatorname{Re}_{D}^{m} \operatorname{Pr}^{n}\left(\frac{X_{n}}{D}\right)^{s}\left(\frac{Z_{n}}{D}\right)^{t}\left[1-\theta_{2} \frac{G(X, I-1)}{G(H, I)}\right]^{u},
$$

where $\mathrm{Nu}_{D}$ is the Nusselt number evaluated with the jet diameter $D$ as the characteristic dimension, $\operatorname{Re}_{D}$ is the
Reynolds number evaluated with the jet diameter $D$ as the characteristic dimension, $\operatorname{Pr}$ is the Prandtl number, $X_{n}$ is the center-to-center nozzle spacing, and $Z_{n}$ is the distance between the nozzle exits and the heated surface. The functions $G(X, I-1)$ and $G(H, I)$ in square brackets describe flow rates per unit area for total impingement and upstream crossflow, $r$ espectively, d ependent o n o n y et f urther geometric/empirical parameters $X, H$ and $I$ which will not be elaborated in detail here as they are not required for the present discussion (but can be found in Ref. [2]). The quantities $\theta_{1}$ and $\theta_{2}$ are correlating constants (values to be determined by comparison with experimental data), while $m, n, s, t$ and $u$ are the exponents of the relevant dimensionless groups in the expression. Note that the first part of Eq. 1 is similar in form to Eq. 5 of our previous paper [1]. Usually the exponents assume constant values in this kind of study (similar to the correlating constants), at least as an approximation in particular regions of flow, pressure, temperature, or particular geometric constraints (normally expressed as ratios).

Amongst other problems with closed-form expressions such as Eq. 1, Kercher and Tabakoff showed that the group $\left(X_{n} / D\right)^{s}$ is not independent but a function of the Reynolds number. In particular, as a "slope parameter" the exponent $s$ varies with Reynolds number. This results in the Buckingham pi theorem being violated. It was concluded that the heat transfer by their square array of impinging jets could not be correlated by power function expressions of dimensionless groups. Interestingly, various authors still attempted such correlations long after the Kercher and Tabakoff publication, probably because that work remained relatively unknown.

Since empirical methods based on dimensional analysis have little chance of success in providing a general description of heat transfer in multi-jet cooling configurations, the only remaining option to interpret measured data appears to be simulations based on CFD.

\section{Measurements}

\subsection{Experimental set-up}

The experimental set-up has been described in detail in our previous paper [1], therefore only some salient points are discussed here. A small circular heated surface $(36 \mathrm{~mm}$ in diameter), a typical beam-spot size on our radionuclide production targets, was supplied by an electrically heated plate. Forced convection of the coolant (air) was supplied by a compressor. Diagrams of this assembly are shown in Figs. 1 and 2, details of which are described below.

A commercial electric element (manufactured by Swift Heat \& Control: $230 \mathrm{~V} \mathrm{AC,} 400 \mathrm{~W}$ ) was embedded into an aluminium plate. The aluminium plate assembly was thermally insulated with mineral wool (supplied by Rockwool International, Denmark) except at the circular gas-cooled surface. The heated surface (circular hot spot) had a diameter of $36 \mathrm{~mm}$. A k-type thermocouple was used to measure the temperature at the solid/gas interface. The power to the heater element could be adjusted with a Variac and Fluke instrumentation was used to read out the voltage and 


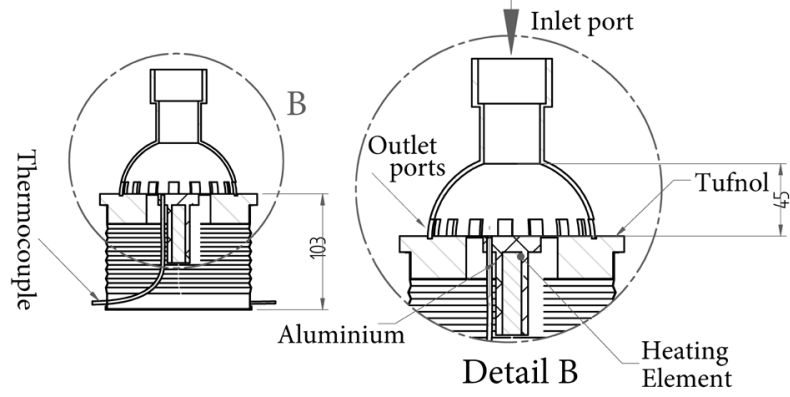

Fig. 1. Cross-sectional views of the electrically heated plate assembly for measuring convective heat-transfer coeffi-cients with multiple air jets. Note the single inlet port at the top and several rectangular outlet ports along the bottom edge of the hemispherical chamber.
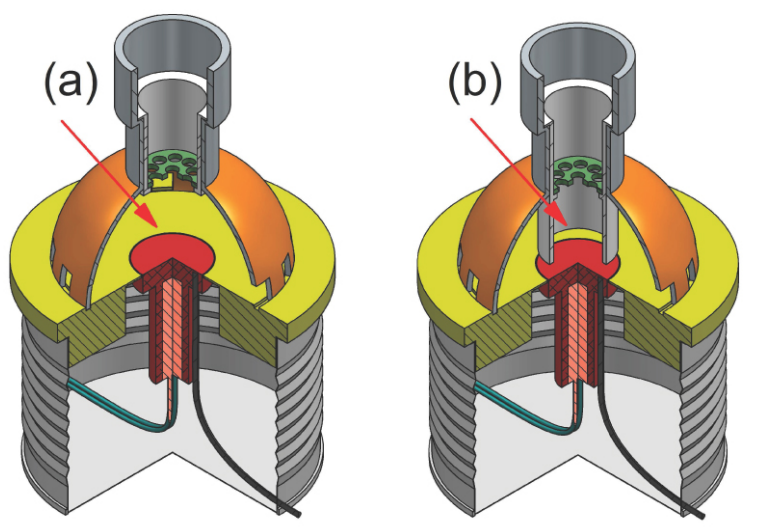

Fig. 2. (a) 3D view of the $90^{\circ}$ hemispherical chamber (or dome) and electrically heated plate assembly with a cut-away to show the heated surface and multi-jet orifice plate. (b) The same view but with a flow guide installed (see text). The jet flow direction is perpendicular to the heated surface and spent gas is exhausted radially. The hot spot is shown in red.

current values. All temperature, voltage and current values could also be streamed to a computer in 1 second intervals. A small chamber with a hemispherical shape (or dome) fitted over the heated surface. The surface area adjacent to the heated surface was made of Tufnol, a material with more than two orders of magnitude lower thermal conductivity than aluminium. Also, the Tufnol plate was machined so that the material in contact with the aluminium plate was only $1 \mathrm{~mm}$ thick. This provided a single hot surface within a region predominantly at room temperature. Rectangular apertures along the bottom edge of the dome served as exit ports for the gas (eighteen exhaust apertures in total, each $10 \mathrm{~mm}$ wide and $11 \mathrm{~mm}$ high, located all around the circumference of the dome). An orifice plate with round holes could be fitted inside the i nlet p ort at the top of the dome to provide the multiple jets (shown in green in Fig. 2).

Five such orifice plates were machined for the experiment, having 1, 3, 7, 10 and 16 holes, respectively. These plates were interchangeable inserts. Essentially, the experimental set-up was the same as in our previous study [1] except that an insert with holes could be fitted at the top of the dome. Each insert had a different number of holes but the total jet cross-sectional area remained the same. These inserts reduced the free flow area considerably compared to the previous single-jet case. The volume flow rate was therefore reduced for these measurements but the bulk linear velocity was nevertheless increased to $50 \mathrm{~m} / \mathrm{s}$ (compared to $36 \mathrm{~m} / \mathrm{s}$ in the case of the single-jet experiment). To be more precise, one of the inserts of the multi-jet experiment also had only one jet: Insert no. 1 (one jet, accurately machined to a diameter of $15.87 \mathrm{~mm}$ ); Insert no. 2 (three jets, machined to a diameter of $9.175 \mathrm{~mm}$ each); Insert no. 3 (seven jets with a diameter of $6 \mathrm{~mm}$ each); Insert no. 4 (ten jets with a diameter of $5 \mathrm{~mm}$ each); Insert no. 5 (sixteen jets with a diameter of $4 \mathrm{~mm}$ each). In order to ensure that the total free flow areas of all the jet inserts were exactly the same, one of the holes had to be machined slightly larger or smaller than the others in a few cases. The idea behind the "flow guide" shown in Fig. 2 (b) will be explained later.

The thermal losses due to mechanisms other than forced convection were determined by covering the heated surface with insulating wool and measuring the electrical power versus temperature characteristics of the system for steady-state conditions without flow. These values were subtracted from the corresponding values obtained with flow. Heat-transfer $r$ ates w ere m easured at $\mathrm{s}$ urface temperatures of nominally $100,150,200,250$ and $300{ }^{\circ} \mathrm{C}$.

\subsection{Results and discussion}

The measured convective heat-transfer coefficients are shown in Fig. 3 as a function of the number of jets. Also shown in Fig. 3 are calculated values according to the Chang, Dittus-Boelter, Sieder-Tate and Petukhov-Kirilov equations for a bulk linear velocity of $50 \mathrm{~m} / \mathrm{s}$. A wall temperature of $300{ }^{\circ} \mathrm{C}$ was adopted for the Sieder-Tate calculation. Similar to the approach in our previous study [1],

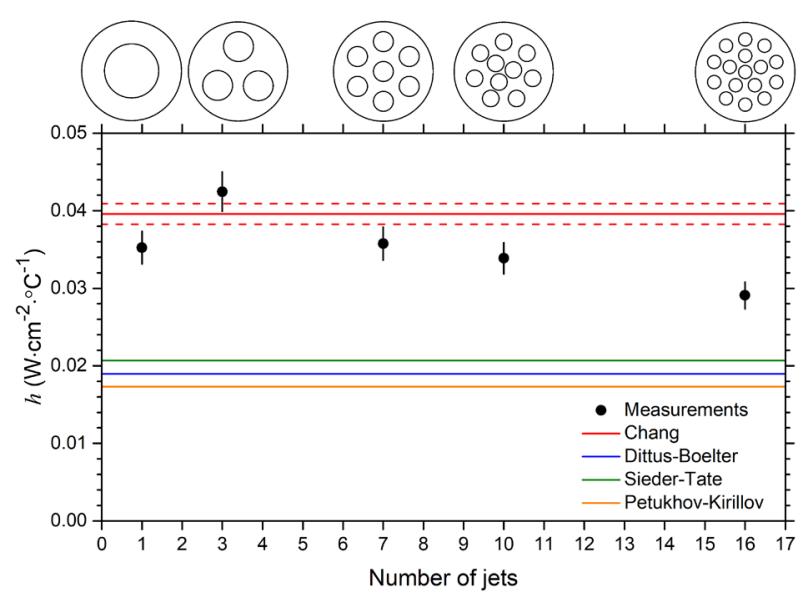

Fig. 3. Convective heat-transfer coefficient $(h)$ plotted versus "number of jets" of multi-jet configuration. The bulk linear velocity of the air was nominally $50 \mathrm{~m} / \mathrm{s}$. Calculated values from other empirical formalisms are also shown, as indicated. The multi-jet inserts are shown schematically at the top of the figure. 
the calculated values are shown as horizontal lines for purposes of comparison with the measured values, however, they are not to be understood as functions of the number of jets. The two red dashed lines are calculations using the Chang equations for flow rates adjusted by $-6 \%$ and $+6 \%$ relative to the nominal value of $35.4 \mathrm{~m}^{3} / \mathrm{h}(50.0 \mathrm{~m} / \mathrm{s})$, respectively, as an indication of the measure of uncertainty. It is important to note that the single-jet Chang formalism was used for the result shown in Fig. 3. The 25-jet equation gives a larger heat-transfer coefficient, in contrast with the trend of these measurements. We concluded that the single-jet Chang formalism proved to be useful in our study but, in contrast, the multi-jet Chang formalism did not provide a useful approximation for the 25 -jet case.

These results are most certainly very case specific. A significant enhancement in the convective heat-transfer coefficient relative to the single-jet value is found only in the 3 -jet case. There is also a very slight enhancement with seven jets relative to one jet, however, this value is reduced considerably relative to the 3 -jet value. This decreasing trend continues towards increasing numbers of jets. As before, the calculations with the Dittus-Boelter, Sieder-Tate and Petukhof-Kirilov equations underestimate the experimental values. The calculation with the singlejet formalism of Chang et al. is much closer to the measured single-jet value but the experimental value is nevertheless lower (Chang single-jet: $h=0.0396 \mathrm{~W} \mathrm{~cm}^{-2}$ ${ }^{\circ} \mathrm{C}^{-1}$; measured single-jet: $h=0.0352 \pm 0.0021 \mathrm{~W} \mathrm{~cm}^{-2}$ ${ }^{\circ} \mathrm{C}^{-1}$ ). The measurement with the three-jet configuration was repeated several times and consistently resulted in higher values than obtained for the single-jet configuration $\left(h=0.0424 \pm 0.0025 \mathrm{~W} \mathrm{~cm}^{-2}{ }^{\circ} \mathrm{C}^{-1}\right)$.

It was speculated by Chang et al. [5] that the increased turbulence caused by the interaction between neigbouring jets may reduce the "jet potential core", leading to reduced stagnation point heat transfer but enhanced heat transfer away from the stagnation points. Based on this notion, there was some speculation at iThemba LABS that perhaps this kind of jet interaction may be having a "diverging" effect on the flow, leading to a significant spreading of the impinging coolant onto the uncooled surface adjacent to the heated surface. Such a spreading would then account for the observed reduction of the convective heat-transfer coefficient with increasing number of jets (for jet numbers larger than 3). In order to investigate this notion, a "flow guide" was installed, shown in Fig. 2 (b). This was just a short section of tubing below the jet insert, extending to $10 \mathrm{~mm}$ above the heated surface. Its purpose was to direct the bulk of the coolant onto the heated surface, preventing the primary jet from straying onto the adjacent uncooled surface. Measurements with the flow guide installed, however, produced nearly the same results as measurements without it. Thus, it was found that the flow guide had no significant effect at all.

One can indeed obtain an enhancement with a multi-jet configuration but, by the same token, one can also obtain a reduction. Consequently, the rule should be to use multiple jets with caution! As empirical formalisms have limited practical value in interpreting multi-jet heat-transfer data, we changed our focus to CFD modelling instead.

\section{CFD modelling}

\subsection{Background and simulations}

In our previous study [1] the code Autodesk CFD 2014 was used to simulate the experimental set-up for single-jet cooling. A later version - Autodesk CFD 2016 - was employed in the present work. The geometry of the set-up, as designed with the drafting code Solid Edge, was imported via AutoCAD Inventor into the CFD code. The coolant flow direction prior to impingement was perpendicular to the plane of the heated surface and a pressure of 1 bar at the exit ports was specified as a boundary condition.

For any CFD simulation involving turbulent flow, an appropriate "Turbulence Model" and "Advection Scheme" must be selected. Here advection refers to the transport of the coolant by bulk motion and, in a general sense, is the combination of convection and diffusion processes. Autodesk CFD 2016 has ten built-in turbulence models and five a dvection $s$ chemes. $T$ his $g$ ives a $r$ ather large number of possibilities, however, it is possible to narrow down the "sensible choices" considerably. From the online instruction manual and other sources, one can evaluate the "recommended uses" of the various turbulence models, e.g. some are recommended for external flows while others are intended for internal flows. O ne can eliminate turbulence models intended for natural convection, lower speed turbulent flows and buoyancy flows, etc. Five of the ten turbulence models are different variants of the Shear Stress Transport (SST) K-omega model. It was found that the standard SST K-omega model was sufficient for our needs, therefore the special versions (namely SAS [Scale Adaptive Simulation]; RC [Rotation and Curvature correction] Smirnov-Menter; RC Hellsten; and DES [Detached Eddy Simulation]) were not required. Together with the K-epsilon model (the default model in the code), it was possible to narrow the turbulence models down to two. The same applies to the advection schemes. Three of them are appropriate for our kind of analysis, namely the Monotone Streamline Upwind scheme, PetrovGalerkin scheme and Modified P etrov-Galerkin scheme (code named ADV 1, ADV 2 and ADV 5, respectively). The Flux Based Scheme (ADV 3) is specially tuned for external flow problems involving significant drag, thus not recommended for the internal flow situation of the present study. Similarly, ADV 4 is specially tuned for flows in long, narrow ducts, thus could be excluded from our investigation. ADV 1 is the default scheme and considered the "work horse" scheme by the developers of the code, recommended as the starting point for most analysis types. ADV 5 is reported to give better accuracy when there are splitting and secondary flows of the coolant, thus one may expect it to be useful in a multi-jet application. It is also reported to be more stable than ADV 2, therefore we chose the modified instead of the standard Petrov-Gelerkin scheme. (Several excellent YouTube videos available on advection schemes and other aspects of the code.)

One of the great strengths of Autodesk CFD is that it is an integrated development environment, which simplifies the w ork fl ow co nsiderably. Th is av oids, for example, mesh generation with a separate pre-processor and 
displaying of the results with a separate post-processor, as is typically the case with most CFD software. In our simulations, Automatic Mesh Sizing was selected to generate the initial mesh. This mesh can be made finer or coarser with a Size Adjustment Slider. The mesh can be viewed in three dimensions by performing rotations, translations, and zooming in and out. Prior to the start of any simulation run, the code performs several checks on the mesh and reports potential problems and/or errors. Other powerful meshing features include Boundary Mesh Enhancement and Enhancement Blending, both of which will be explained in more detail below.

In our previous study on single-jet gas cooling [1], the SST K-omega model was found to give results in better agreement with experimental data than the default K-epsilon model. In the present study, convergence issues/errors were sometimes experienced with the K-epsilon model, especially with increasing numbers of jets. These problems were not found with the SST $\mathrm{K}$-omega model. Consequently, we decided quite early in the investigation to discontinue using the K-epsilon model. Finally, only the SST K-omega model in conjunction with the ADV 1 an ADV 5 advection schemes were retained for our analysis, reducing the 50 possible combinations of "turbulence model + advection scheme" to only two.

In the SST K-omega model, no "damping functions" or "wall functions" are used to describe the strong attenuation of the normal component of turbulence near the wall boundary, as is done in the K-epsilon model. Instead, the turbulence is simulated all the way to the wall, requiring the mesh to be very fine in the boundary layer region. The default number of mesh layers at a wall boundary is 3 but it is recommended to increase this number when using the SST K-omega model. This is done by using the Boundary Mesh Enhancement feature of the code. The 2014 version of the code allowed the number of boundary mesh layers to be increased to 10 , while the 2016 version allows up to 15 layers. The price to pay for increasing the number of layers is a large increase in the total number of elements and the simulation becoming much more resource intensive, requiring a significantly longer execution time. In order to keep the total number of elements below $3 \times 10^{6}$ in the case of 16 jets, the number of mesh layers at the wall was increased from 3 to only 8 . While 10 layers is normally recommended for the SST K-omega model, it was found that there was not much difference in the results by adopting a parameter value of 8 . With a top-end core-i7 $\mathrm{PC}$, this choice would result in the completion of a run in under 12 hours, therefore a second run could be started on the same day and completed during the night. The number of elements and the execution time increased with increasing number of jets. The total number of elements varied between about 800000 for the single-jet case to $3 \times 10^{6}$ for the 16-jet case.

Enhancement Blending was selected in all our simulations. The code default is with Enhancement Blending disabled, which results in an instantaneous transition from a highly anisotropic wall region to an isotropic interior region. A more gradual transition in element size between wall regions and interior regions is obtained when
Enhancement Blending is enabled, which is often recommended for simulations with the SST K-omega models.

The boundary conditions were as follows: Inlet air temperature $-20^{\circ} \mathrm{C}$; Outlet air pressure - 1 bar; Bulk flow rate - $35.4 \mathrm{~m}^{3} / \mathrm{h}$. The default convergence criterion is halfway between "loose" and "tight" in the code, with a slider to select different values. The notes in the code recommend tightening the convergence criteria once a new problem runs well. The slider was typically adjusted from the $50 \%$ position to the $80 \%$ position, i.e. towards a tighter convergence criterion. This was found to be a good compromise. With the tightest convergence criterion (slider at $100 \%$ ) some simulations took significantly longer to complete. With CFD simulations, refinements usually have a price to pay in terms of extended execution time.

\subsection{Results and discussion}

The measured convective heat-transfer coefficients are compared with the corresponding CFD results in Fig. 4. In the cases of single-jet and 3-jet cooling, the results obtained with the Monotone Streamline Upwind scheme (i.e. the ADV 1 advection scheme) are in very good agreement with the measurements but towards an increasing number of jets one observes an underestimation. In contrast, the Modified Petrov-Galerkin scheme (i.e. the ADV 5 advection scheme) produces an overestimation for the single-jet and 3-jet heat transfer but good agreement with the experimental values for the 7 -jet, 10 -jet and 16 -jet cases. In Fig. 4, the closest CFD results to the measurements have been filled with magenta colour. Indeed, the magenta symbols show good agreement with the experimental values. This is very interesting because, from the perspective of the fluid flow, an increase in the nu mber of je ts le ads to

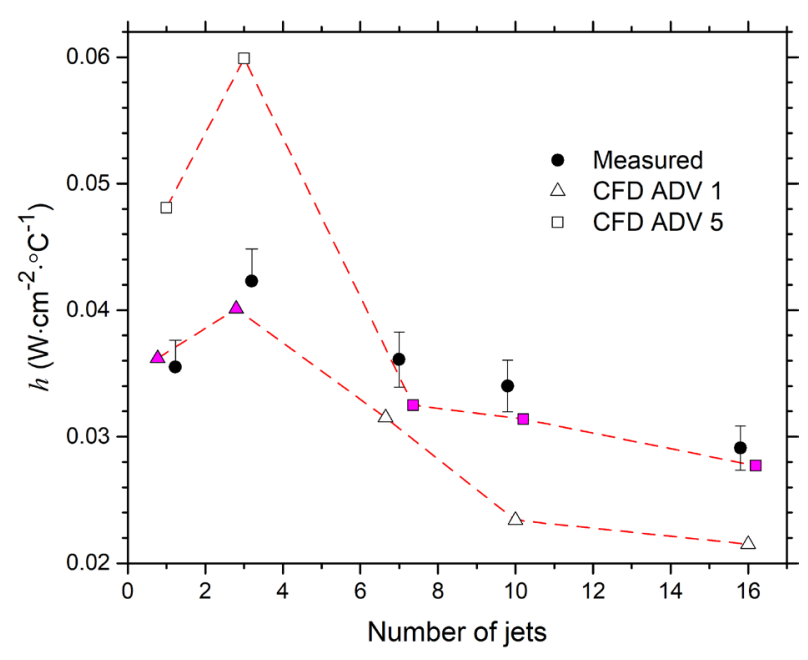

Fig. 4. Convective heat-transfer coefficient versus number of jets. The measured values are compared with CFD results obtained using two different advection schemes, as indicated (see text). Note that some results are slightly displaced on the $\mathrm{x}$-axis to prevent overlap of the symbols. The CFD results closest to the measured values are indicted with a magenta fill. The dashed lines are to guide the eye. 
more complexity in the flow in terms of separation and recombination. The ADV 5 advection scheme is reported to be superior in such cases. In the simpler cases of singlejet and 3-jet flow, however, the ADV 1 advection scheme gives better agreement. In the case of 7 jets, ADV 1 and ADV 5 give very similar results. Whether this is truly significant or simply a coincidence is debatable, however, it is not unexpected that ADV 5 should give better results in cases where separation and recombination in the coolant become more abundant. It is not clear to us whether one should expect ADV 1 to be superior in the cases of simpler flows, however, in this particular study such a result was indeed obtained. Both sets of CFD simulations exhibit a maximum heat-transfer coefficient with 3 jets, in agreement with the experimental results.

The above results were obtained with thin ( $3 \mathrm{~mm}$ thick) orifice plates to form the jets. In many applications, however, jet nozzles are rather elongated in shape. We asked ourselves whether jet length would play a significant role. We therefore performed a set of CFD simulations with elongated jets. The nozzle length was increased to $100 \mathrm{~mm}$ (i.e. many jet diameters in length for all cases), thus one would expect the flow to become fully developed in a jet prior to reaching the nozzle exit. These thick nozzle inserts extended upstream into the tube through which the coolant entered the dome. This is evident in Fig. 5, which shows a shaded wire view of the fluid mesh for the 7-jet case (note that all solid structures were made invisible in this view in order to show only the fluid.) Figure 6 compares the CFD results obtained with thin jet inserts and thick jet inserts. It is evident that the nozzle length has only a minor effect on the results. Note that ADV 5 was used in these simulations. It is interesting that slightly higher heat-transfer coefficients have been obtained with the thinner nozzles but the reason for this is not clear.

Next, we questioned the effect of the distance of the nozzle exits to the heated surface. CFD simulations were therefore also performed at two smaller nozzle-to-heated-

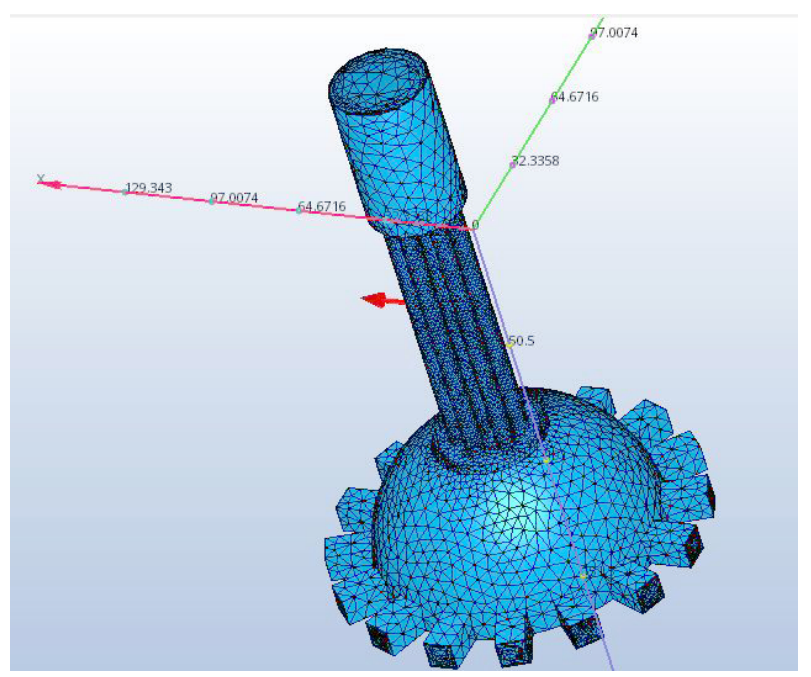

Fig. 5. Shaded wire view of the fluid mesh in the 7-jet assembly with elongated jets.

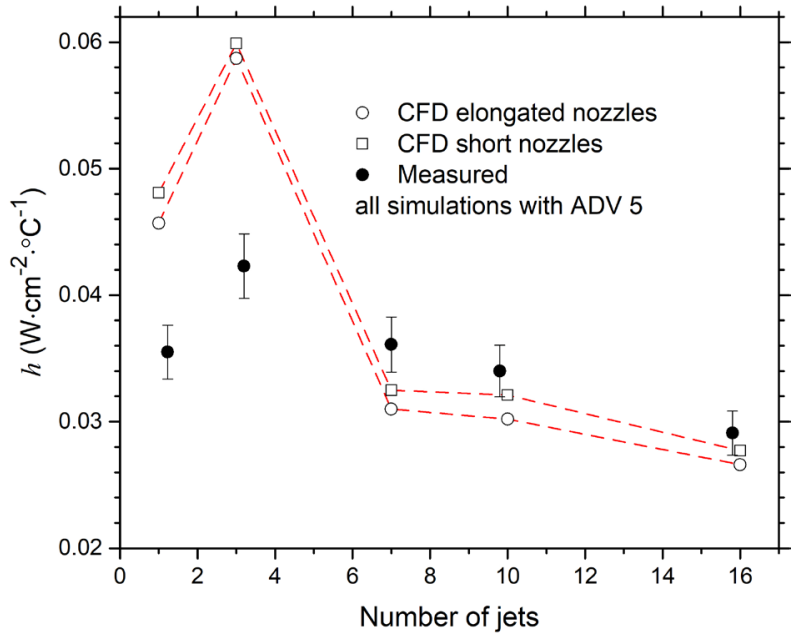

Fig. 6. Convective heat-transfer coefficient versus number of jets. The CFD results with elongated nozzles $(100 \mathrm{~mm})$ are com-pared with CFD results obtained with a thin $(3 \mathrm{~mm})$ nozzle insert. The dashed lines are to guide the eye. The measured values (ob-tained with thin nozzles) are also shown for comparison.

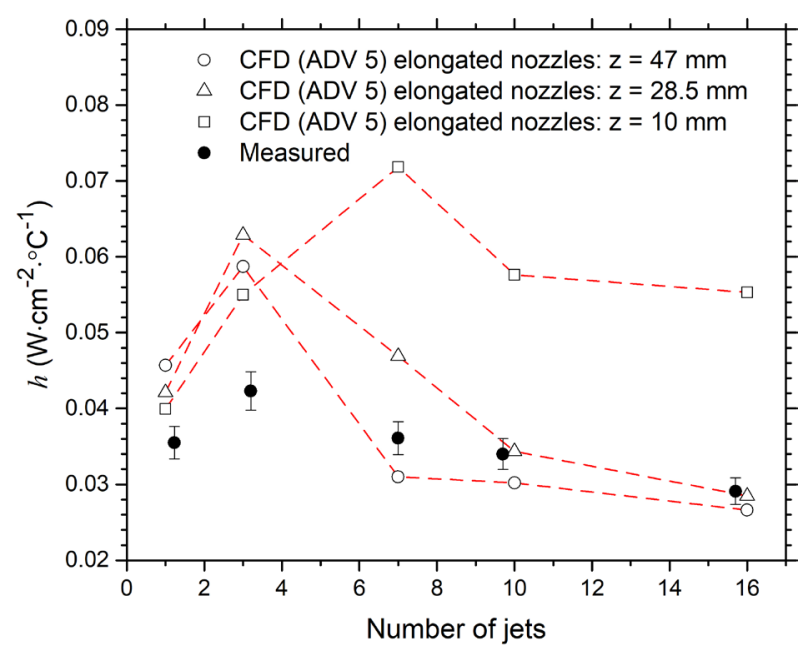

Fig. 7. Convective heat-transfer coefficient versus number of jets. The CFD results with elongated nozzles $(100 \mathrm{~mm})$ are compared at three nozzle-to-heated-surface distances, as indicated. The dashed lines are to guide the eye.

plate distances to investigate this. The three sets are compared in Fig. 7. Note that advection scheme ADV 5 was again selected for this comparison. It is clear that the nozzle-to-heated-plate spacing has a significant effect on the results. It seems generally advantageous to place the nozzles closer to the heated surface. At the smallest nozzle-to-wall separation $(10 \mathrm{~mm})$ the heat-transfer coefficient was found to be larger in the 7-jet c ase. Thus, the maximum shifted from the 3 -jet to the 7-jet configuration by placing the jets closer to the surface.

In the final set of simulations performed, the flow rate was increased from $35.4 \mathrm{~m}^{3} / \mathrm{h}$ to $100 \mathrm{~m}^{3} / \mathrm{h}$. The results are shown in Fig. 8. As expected, higher heat-transfer coefficients have been obtained with the higher flow rate, however, the trend of the heat-transfer coefficient with respect 
to number of jets remained the same. The difference between the two sets of results seems to be a simple scaling factor, to a good approximation.

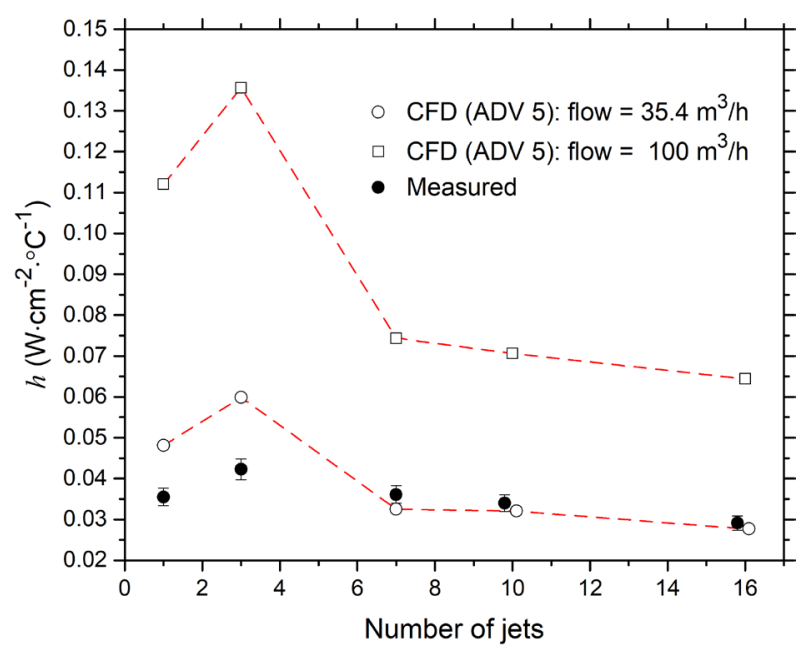

Fig. 8. Convective heat-transfer coefficient versus number of jets. The CFD results with elongated nozzles (100 $\mathrm{mm}$ in length) are compared at a nozzle-to-heated-surface distance of $z=47$ $\mathrm{mm}$ but for two different flow rates, as indicated.

\section{Conclusion}

It has been shown that the use of multiple jets can enhance turbulence, hence the convective heat-transfer coefficient. Whereas Chang and co-workers found an enhancement with a $5 \times 5$ square array with respect to their single-jet case, in our experiment we found an enhancement with respect to the single-jet case only with our 3-jet and 7-jet configurations, whereafter the further increase of the number of jets lead to a monotonically decreasing heattransfer coefficient, and a worsening relative to the singlejet case. Geometry clearly plays an important role, making it very difficult to use dimensional analysis to relate the heat transfer to the various hydrodynamic properties. In this regard, the cross flow of spent gas towards the exhaust ports play a major role. Due to these complexities, CFD simulations in conjunction with experiments seem to be the only alternative to study the behavior of such systems.

In this study, which should be seen as a continuation of our previous study reported at INTDS 2016, we endeavoured to utilize multi-jets as a way to improve the cooling of window foils on targets and beamlines with gas as the coolant. Since the flow in s ubmerged m ulti-jet systems is complex and non-intuitive, one can easily get it wrong. Turbulence enhancement is indeed possible but one can also achieve the opposite, i.e. a reduction in turbulence in the region where most of the heat transfer takes place.

The SST K-omega model, within the context of CFD simulations, shows good potential as a means to study multi-jet cooling of small heated objects. It provided convective heat-transfer coefficients in good agreement with the experimental results of this study but there is a clear dependence on the choice of advection scheme. The Modified Petrov-Galerkin scheme provided good agreement in cases where the number of jets is higher (more than 3 ). In the simpler single-jet and 3-jet configurations, very good agreement was obtained with the Monotone Streamline Upwind scheme. The latter advection scheme seems to be better for simple flow geometries while the former one better for more complex, separated flows.

Multi-jet cooling in targetry clearly has potential but must be implemented with care. There are no "rules of thumb" or readily available approximations. A combination of experimental investigation and CFD modelling is required to gain a better understanding of the heat transfer.

Lastly, we need to point out that our measurements were performed using air as coolant. This was done for the sake of ease and also because the assembly was not helium tight. Normally, helium would be used as it is superior to air as a coolant gas, in addition to not forming problematic activation products.

This work was financially supported by the National Research Foundation (NRF Grant no. 85507).

\section{References}

[1] G.F. Steyn, C. Vermeulen, INTDS2016, AIP Conf. Proc. 1962 (2018) 030020-1 - 030020-11

[2] D.M. Kercher, W. Tabakoff, J. Eng. Power 92 (1970) 73-82

[3] H. Martin, Adv. Heat Transfer 13 (1977) 1-60

[4] Y. Shan, J. Zhang and G. Xie, Int. J. Heat Mass Transfer 86 (2015) 832-842

[5] C.T. Chang, G. Kojasoy, F. Landis and S. Downing, Int. J. Heat Mass Transfer 38 (1995) 833-842

[6] H.Q. Yang, T. Kim, T.J. Lu and K. Ichimiya, Int. J. Heat Mass Transfer 53 (2010) 4092-4100

[7] K. Nanan, K. Wongcharee, C. Nuntadusit and S. Eiamsa-ard, Int. Com. Heat Mass Transfer 39 (2012) 844-852

[8] Y. Yu, J. Zhang and H. Xu, Int. J. Heat Mass Transfer, 72 (2014) 222-233 